also the values of the slope at the origin, $\left.\frac{d B}{d H}\right]_{0}$, respectively, have been plotted as a function of the diameter of the wire. In this way the two curves shown in the accompanying graph have been obtained.

Each of the curves has a well-defined maximum value, and the general character of each curve can be accounted for if it be assumed that: (i) the wire has a magnetically hard surface layer; (ii) the process of magnetization commences at the surface of the wire and proceeds progressively towards the axis by a kind of 'chain-effect', so that the magnetic induction is greatest near the surface and progressively decreases towards the axis of the wire.

Further tests are being carried out at higher temperatures, and a full account of the results will be published elsewhere in due course.

$$
\text { T. F. WALL. }
$$

Department of Electrical Engineering,

The University,

Sheffield, 1.

Oct. 3.

1 NATURE, 141, 205 (Jan. 29, 1938); also The Engineer, June 24, 1938.

\section{Diffraction Patterns Produced by Surfaces in Sunlight}

The reflection of sunlight from various surfaces, including those of finger nails, skin, paper, wood, plant leaves, and glass slides covered with thin films of dried blood, produces mosaic-like varicoloured patterns which yield to the unaided eye an impression of the presence of many small but discrete coloured granular particles. The coloured mosaics are particularly brilliant when the surfaces are viowed near grazing incidence, although in some instances the effect is clearly evident from other angles. Such patterns are not evident on the polished surface of glass. The optical irregularity of the former surfaces is commonly evident to the unajded eye only by the generalized cloudiness or haziness. The mosaic pattern is readily seen when the reflecting surface, adjusted to an appropriate angle, is placed at a distance of approximately four to fourteen inches from the eye. At greater distances the detail of design is lost. At lesser distances the chromogenic features are not apparent. An illusion of flow of the coloured 'particles' is produced by slight tilting of the surface while under observation. It thus appears as if different groups of surface projections come into play with a resultant rearrangement of the coloured spots.

The production of coloured patterns by reflection of white light from surfaces is a commonly recognized phenomenon. However, the mosaic effect produced by the above-mentioned surfaces is different, in appearance at least, from those which have been described previously. Among the well-known colour effects produced by reflection are the colours of striated surfaces and the colours of fine laminar structure. The former, usually seen as a pattern of concentric rings upon the finely scratched surface of glass and metal, was attributed by Tyndall ${ }^{1}$ to interference of light reflected from the opposite sides of the furrows or seratches. The coloured areas seen in certain shells and pearls are associated with fine laminar structure and are attributed to the class of phenomena associated with Newton's rings. There is a conceivable similarity of the colours of the mosaic to those produced by reflection of light through multi-layers of surface films spread on metal plates $^{2}$, except that in the former instance the areas of a given thickness are extremely small and the reflecting foci are tilted at different angles; hence the mosaic-like effect.

The fact that the mosaic effect appears in sunlight and in light from distant incandescent lamps, while not in light originating from a nearby source, suggests that the phenomenon is to be classed with the Fraunhofer diffraction phenomena ${ }^{3}$.

The mosaic pattern is apparently a consequence of the mutual interference of diffraction bands produced when parallel light rays strike the small component parts of certain surfaces. This regularity of the chromogenic interference mosaic for certain materials provides additional means of characterizing the structure of such surfaces.

C. Wesler scull.

\author{
Abington Memorial Hospital, \\ Abington, Montgomery County, \\ Pennsylvania. \\ Sept. 9. \\ 1 Tyndall, J., "Lectures on Light" (D. Appleton and Co., N.Y., 1873). \\ "Langmuir, I., Schaefer, V. J., "Built-up Films of Proteins and their \\ Properties", Science, 85, 7̈6 (Jan. 15, 1937). \\ "Wood, R. W., "Physical Optics" (new and revised edition; The
} Macmillan, Co., New York, 1929).

\section{Cause of Earthquake Ruptures}

As present there is no established theory of the cause of earthquake ruptures because of their diversity. Observations in Cordoba of the San Luis shocks of May 1936, including records on a sensitive Richard barograph at the National Observatory, have suggested that this diversity of behaviour is due chiefly to differences of depth, of dip and strike of the faults, and that barometric pressure is often the final actuating cause which determines the hour and perhaps the day.

Actual eracks observed in the surface in many places confirm that the underlying conditions in the tectonic type of earthquake is an accumulation of strains along geological faults in the earth's crust, the sudden release of which produces the shock. Whatever the position and direction of these strains, the gravitational pressure of the masses must tend to hold the surfaces together. Without gravitational pressure, no strain could accumulate. A sufficient release of such pressure will cause a rupture. Confirmation of this is found in the cases where shocks have occurred chiefly at low tide.

Tidal stresses caused by the sun and moon are alone capable of effectively decreasing these pressures. Nearly all extensive series of observations have disclosed a slight preference for times of new and full moon. The San Luis earthquakes referred to suggested that in deep shocks, where the gravitional pressure of the mass above was greatest, the connexion was closer than in shocks nearer the surface.

To test this point, the most destructive shocks, fifty-nine in number, in the past three centuries, for which data could be found, and forty heavy shocks reported in the past two years, were examined. In both these groups, approximately eighty per cent showed preference for the times of new and full moon.

These same shocks showed a preference for the times of lunar perigee. Extensive series of after-shocks 